\title{
Mining Movies to Extract Song Sequences
}

\author{
Sher Muhammad Doudpota* \\ CSIM, Asian Institute of Technology \\ Bangkok, Thailand \\ sher.muhammad.doudpota@ait.ac.th
}

\author{
Sumanta Guha \\ CSIM, Asian Institute of Technology \\ Bangkok, Thailand \\ guha@ait.ac.th
}

\begin{abstract}
This paper proposes a system to automatically locate and extract songs from digitized movies. We focus on the genre of Bollywood movies. A song grammar particularly applicable to this genre is proposed and used subsequently to construct a probabilistic timed automaton to differentiate songs. The proposed system has been implemented and test results indicate both high precision and recall. Songs being a major driver in the success of Bollywood movies, a potentially significant application of the proposed system lies in automatically mining the vast Bollywood movie archives.
\end{abstract}

\section{Keywords}

Song Extraction, Movie Indexing, Probabilistic Timed Automata, Scene Detection, Event Detection

\section{INTRODUCTION}

The Indian film industry, particularly the segment of the industry, commonly known as Bollywood, which produces Hindi language movies, enjoys a wide following in South Asia, the Middle East, Central Asia, North Africa and in countries, such as the UK and USA, where there is a significant South Asian diaspora. In fact, the Indian film industry, which includes, in addition to Bollywood, regional segments producing movies in local languages like Telugu, Bengali, Malayalam, and Kannada, is the most prolific in the world. In 2009, it produced 2961 movies including 1288 feature films[4]

Bollywood has become an important part of South Asian culture. The most common Bollywood genre, the song-anddance movie, colloquially called the "masala" movie, typically with a simple melodramatic plot along the lines of "poor good guy wins the rich beautiful gal", resonates strongly

*Sher Muhammad Doudpota is currently a PhD student at CSIM, Asian Institute of Technology, Thailand

${ }^{\dagger}$ Dr Guha is an Associate Professor at CSIM, Asian Institute of Technology, Thailand

Permission to make digital or hard copies of all or part of this work for personal or classroom use is granted without fee provided that copies are not made or distributed for profit or commercial advantage and that copies bear this notice and the full citation on the first page. To copy otherwise, to republish, to post on servers or to redistribute to lists, requires prior specific permission and/or a fee.

MDMKDD'11, August 21, 2011, San Diego, CA, USA.

Copyright 2011 ACM 978-1-4503-0841-0. across the subcontinent. Often derided as escapist fare, Bollywood movies, nevertheless, are hugely popular amongst all strata of South Asian society, from daily wage workers to millionaire industrialists, from the uneducated to the collegegoing. Families gathering around the TV at home to watch a Bollywood movie is a favorite leisure pastime in South Asia. South Asians abroad, in fact, are often even more fanatical in collecting and viewing Bollywood movies, an activity which transports them, at least vicariously, back to their homeland. Popular film songs often form the musical backdrop for various festive occasions in South Asia, from weddings to political rallies.

Not surprisingly, songs are a crucial determinant of a Bollywood movie's success. In fact, a main purpose of the plot line is to provide a sequence of pegs on which to hang song sequences. There are usually three to ten songs in a Bollywood movie of two to three hours, with every song having length of three to ten minutes. The revenue generated by the music and songs of a movie is around $4-5 \%$ total of the total. The importance of film music and songs can be realized as well from the fact that $48 \%$ of India's music sales are from film music.

The popularity of Bollywood movies songs has led to the growth of a separate industry to produce cassettes and CDs containing only song clips from Bollywood movies. These CDs and cassettes sell in huge volumes throughout South Asia and other countries where Bollywood movies are popular. There are online databases as well from which Bollywood songs can be downloaded. Now, that even Bollywood movies from the pre-digital era of film on reels have mostly been digitized, there is huge virtual archive of such movies, with a commercial need for extracting the song sequences. Manual extraction is obviously a time-consuming and errorprone task. Evidently, therefore, a system to automatically extract song sequences from digitized Bollywood movies, as we propose, should be of practical interest.

A problem related to music and song extraction is automatic scene detection. A scene in this case refers to dialogue or action scene. There are many recent attempts to extract a particular type of scene from movies. An audiovisual-based approach for scene indexing is presented by Lehane et al. [2]. The proposed approach considers three classes of scenes: dialogue, action, and montages. The montages include music sequences. The authors apply film making principles and finite state machines to detect the occurrences of these 
three types of scenes. They propose a complete movie search system, called MovieBrowser, which can help a viewer reach a desired scene directly. When applied to a 23-hour long movie video, they achieved $84 \%, 93 \%$, and $81 \%$ accuracy for dialogue, action, and montage scenes respectively. However, according to their definition, a montage scene does not necessarily mean just songs; it can be any scene having background music. For example, shots of somebody crying, a romantic sequence of shots and a musician playing at a funeral are all considered montages. Therefore, their scene detection system is not enough for our purpose of isolating only song sequences.

Lei Chen et al. in [10] present a top-down approach which uses video editing rules and audio cues to extract dialogue and action scenes. They use a finite state machine with an audio-based SVM (support vector machine) classifier for detection of scene type. Their proposed classifier uses three features of movie audio including variance of zero crossing rate, silence ratio, and harmonic ratio. They achieved a precision of $76.56 \%$ and recall of $81.6 \%$.

In another attempt to extract dialogue scenes from a movie, Bart Lehane et al. [3] use low and mid-level visual features for characterizing individual shots, which are then combined to form a meaningful dialogue scene. They propose a threetier system. At the lowest tier they perform shot boundary detection along with motion extraction. The extracted shot boundary and motion are used at the next tier for shot clustering. Finally, at the highest tier the clustered shots are fed into a finite state machine to extract meaningful dialogue scenes from the video. When applied to six popular Hollywood movies, the authors achieved recall and precision of $77.8 \%$ and $86 \%$, respectively.

Other attempts at indexing movies [12, 14, 9] have considered mainly action and dialog scenes. They rarely consider music and songs as separate units of a movie. However, for Bollywood movies this distinction must be made.

In this paper, we use general song making principles and propose a finite automaton based model for locating and extracting songs from Bollywood movies automatically. Our approach towards song extraction is based on two steps: in the first step, we use an SVM classifier to classify the input movie into music and non-music classes using three audio feature: zero crossing rate, spectrum flux, and short time energy and sub-band energy distribution. In the second step, we apply song making rules and an analysis of song features to construct a probabilistic timed automaton for classification between song and non-song scenes.

The rest of the paper is organized as follows. Section 2 gives an overview of our proposed system. Section 3 discusses how the music part of a movie is separated from the nonmusic part using an SVM classifier. The process of extracting songs from the music part is explained in the section 4 . The section 5 presents a pattern checker that checks pattern of the extracted songs to make sure that unwanted action/dialogue scenes are filtered out from final output. Experimental results are presented in the section 6 . Finally, the section 7 concludes the paper.

\section{SYSTEM OVERVIEW}

The abstract model of the proposed system for song extraction from movies is shown in Figure 1. It comprises of two building blocks: an audio classifier and a song extractor. The role of the audio classifier is to classify the input movie contents into music and non-music segments. The non-musical part of a movie, where mostly dialogue is found, is discarded. Songs, on the other hand, are found in the musical part of the movie, which is the input to the song extractor in our system.

The first component of a song extractor is a potential song sequence generator (PSSG). This component uses a few basic song making rules to generate potential song sequences (PSS). A PSS is highly likely to be a song; however, it may also be an action or dialogue scene. We expand on this later in the song extractor section. The next component in a song extractor is the vocal detector. The job of the vocal detector is to identify vocal and non-vocal parts in a potential song sequence. A vocal in our definition refers to either a singing voice or human speech over music, whereas a nonvocal refers to a music-only part in a potential song sequence. Finally, the pattern checker component of the song extractor checks the vocal and non-vocal pattern to decide if a potential song sequence is either a song or a dialogue/action scene.

\section{AUDIO CLASSIFIER}

A Bollywood movie's song can be defined as a male or female singing voice over music. The fact that every song is accompanied by music, leads us to search for songs in the music part of a movie. Thus, the first component of the proposed system is an audio classifier that classifies an input movie contents into music and non-music classes.

There are many attempts in the literature to classify audio into music and non-music classes $[7,11,8,15,13,14,16]$. Our approach is similar to that of Lu et al. [14]. The steps involved in the music vs. non-music classification are illustrated in Algorithm 1 . The first step (line 1) is to break the input movie into frames to form a window size of one second. The window size is set to one second because it seems to give the best performance. The performance of the audio classification depends mainly on the selection of appropriate audio features and classifier. In step two (line 2) we extract audio features including zero crossing rate, short time energy/sub-band energy distribution and spectrum flux from each frame of a 1-second window. These features are further explained in the next section. In the third step (line 3), an SVM classifier is used to classify each frame as either music or non-music based on audio features. We use an SVM classifier because it performs well on binary classification as in our problem. Finally, in step four (line 4), the algorithm returns music and non-music segments of the input movie. The music segment is further used for song extraction in the next component of the system.

\subsection{Zero Crossing Rate}

The frequency contents of an audio signal can be measured by zero crossing rate. It is defined as the number of time-domain zero crossings within a frame:

$$
Z C R=\frac{1}{2(N-1)} \sum_{m=1}^{N-1}|\operatorname{sgn}[x(m+1)]-\operatorname{sgn}[x(m)]|
$$




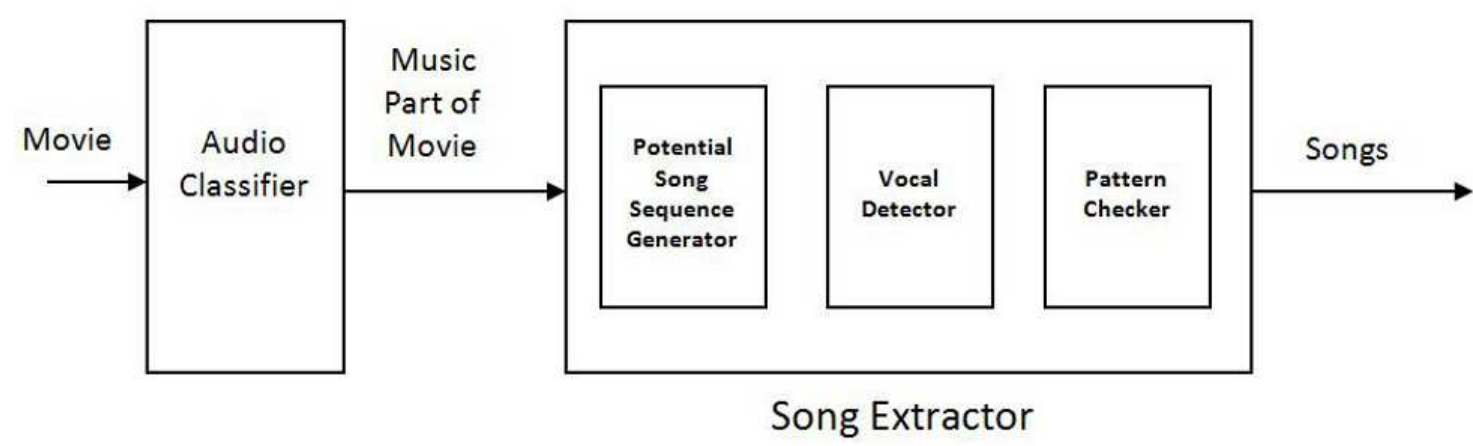

Figure 1: Abstract Model of Song Extractor

Input : Movie $m$, Frame length $l$

Output: Music and non-music segments of movie $m$

1 Segment movie $m$ into frames with length $l$;

2 Calculate audio features for each frame;

3 Classify movie frames in music and non-music classes using Support Vector Machine;

4 Return music and non-music segments;

Algorithm 1: Music Non-music Classification

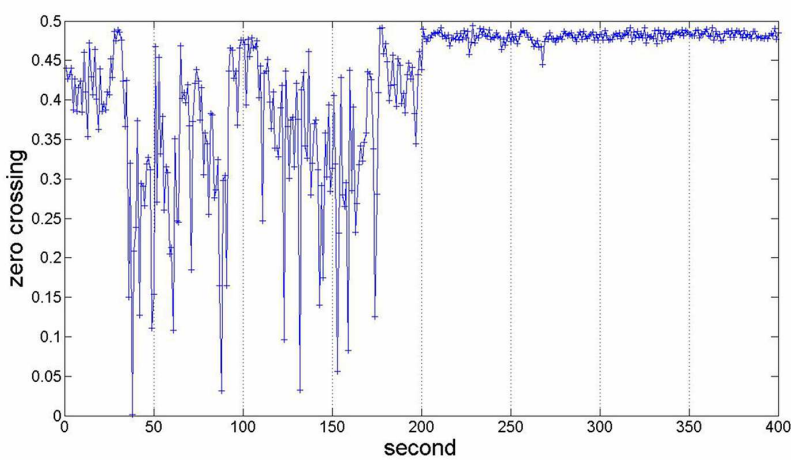

Figure 2: Zero Crossing of Speech and Music Audio Signal

where $x(m)$ is a discrete audio signal and $\operatorname{sgn}[]$ represents a sign function[14].

The speech signal (without music) in an audio contains silent parts (breaks between speech words) that cause higher zero crossing variations in comparison to that of a music signal; thus, ZCR is an efficient discriminator between music and speech. We show the difference between $Z C R$ variations of a music and a speech signal in Figure 2. In Figure 2, the speech ZCR are shown from second 1 to 200, whereas the music ZCR are shown from second 200 to 400 . It can clearly be seen that zero crossing variations during the speech signal are much higher than the music signal.

\subsection{Spectrum Flux}

The spectrum flux is defined as the average variation value of the spectrum between the adjacent two frames in an audio clip [14]. It is defined as,

$$
\begin{aligned}
S F= & \frac{1}{(N-1)(K-1)} \times \\
& \sum_{n=1}^{N-1} \sum_{k=1}^{K-1}[\log (A(n, k)+\delta)-\log (A(n-1, k)+\delta)]^{2}
\end{aligned}
$$

where

$$
A(n, k)=\left|\sum_{m=-\infty}^{\infty} x(m) w(n L-m) e^{j \frac{2 \pi}{L} k m}\right|
$$

and $x(m)$ is the input discrete audio signal and $w(m)$ the window function, $L$ is the window length, $K$ is the order of DFT, $\delta$ is a very small value to avoid calculation overflow and $N$ is total number of frames in an audio clip.

Generally, the spectrum flux is the spectrum value difference between two adjacent frames. This difference is higher in speech signals because speech consist of voice and silent (breaks between speech) parts, causing abrupt changes in spectrum values. On other hand, the music signal is more consistent than speech with less spectrum difference between adjacent frames. In order to verify this statement we performed an experiment to show the difference between the SF of speech and music. Figure 3 shows the SF of the music signal from second 1 to 200, whereas 201to 400 is SF of an speech signal.

\subsection{Short Time Energy and sub-band Energy Distribution}

The short time energy represents the total power spectrum of a frame. It is defined as,

$$
S T E=\log \left(\int_{0}^{w_{0}}|F(x)|^{2} \mathrm{~d} w .\right)
$$

where $F(w)$ denotes the Fast Fourier Transform coefficients, $|F(w)|^{2}$ is power at frequency $w$ and $w_{0}$ is half sampling frequency [14].

The STE or power spectrum variations in the speech signal are again found higher in comparison to the music signal due to silent breaks in the speech signal. Figure 4 shows 


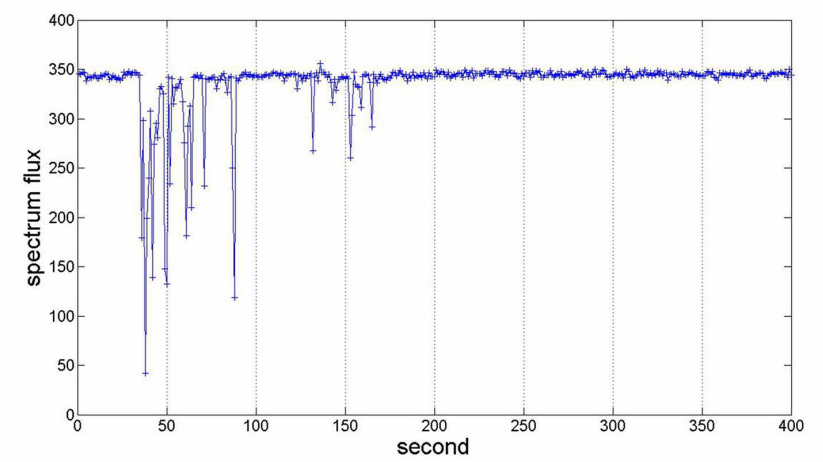

Figure 3: Spectrum Flux Music and Speech Audio Signal

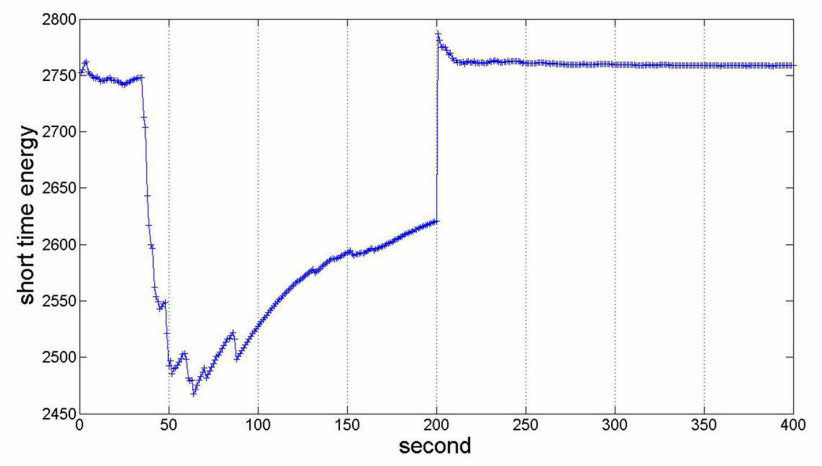

Figure 4: Power Spectrum/Short Time Energy of Music and Speech Audio Signal

power spectrum of the music from second 1 to 200 and power spectrum of the speech from second 201 to 400.

\section{SONG EXTRACTOR}

The song extractor in our system accepts the music part of an input movie from the audio classifier and searches for song sequences in it. The music part of a movie certainly contains the movie's song, but there could also be dialogue and action scenes with background music in this part. Thus, we need a mechanism to filter out dialogue and action scenes, retaining only songs. In order to accomplish its task the song extractor is divided in three sub-components: potential song sequence generator, vocal detector, and pattern checker. We now explain the working of these individual components.

\subsection{Potential Song Sequence Generator}

A potential song sequence generator accepts the music part of a movie and generates a number of potential song sequences (PSS), which are highly likely to be songs. We observed all 74 songs of 10 movies, selected for the experiment. None of the songs is shorter than 120 seconds in duration. The potential song sequence generator uses this fact to form its rules for PSS generation. It first breaks the music part of a movie into multiple sequences where a sequence refers to consecutive musical seconds without break. It then applies the following rules of sequences in order to extract a potential song sequence,
Rule 1: Check the length of a sequence: if it is greater than or equal to 120, extract it as a potential song sequence (PSS); otherwise, apply rule 2 .

Rule 2: If the length of a sequence is not equal to or greater than 120, check the difference between last second of the current sequence and the first second of the next sequence. If the difference is less than or equal to 3, combine both the sequences and check the length of combined sequence. If the combined sequence length is greater than or equal 120, extract it as a potential song sequence; otherwise, discard current sequence and check next sequence.

The difference check in Rule 2 is motivated in order to account for possibly inaccurate classification. Particularly, the audio classifier might misclassify a musical second as non-musical, causing a potential song sequence to be discarded.

The PSS generated by the PSSG are highly likely songs. However, in certain cases, there might also be dialogue or action scenes with background music having length equal to or greater than 120 seconds. Such action/dialogue scenes would also be generated as PSS by the PSSG. We need a mechanism to differentiate the PSSs that represent song from PSSs that represent action/dialogue scene.

This differentiation can be done by analyzing the vocal (human voice over music) and non-vocal (music-only) pattern in a PSS. A song follows a certain pattern of vocal/non-vocal, whereas in action/dialogue scenes no such fixed pattern is followed. In order to check the song pattern in a PSS, we need to identify vocal and non-vocal seconds in a PSS. The next section describes our vocal detector.

\subsection{Vocal Detector}

Our approach towards vocal/non-vocal classification is similar to that of the audio classifier described earlier. However, along with the features for music/non-music classification, two additional features, spectral centroid and mel-frequency cepstral coefficients are used for vocal/non-vocal classification. These are explained below.

\subsubsection{Mel-frequency Cepstral Coefficients}

The mel-frequency cepstral coefficients (MFCC) are commonly used in speech recognition. They are a short term power spectrum representation of a sound and defined as[14],

$$
c_{n}=\sqrt{\frac{2}{K}} \sum_{k=1}^{K}\left(\log S_{k}\right) \cos [n(k-0.5) \pi / K] \quad n=1,2, \ldots L
$$

where $K$ is the number of band pass filters, $S_{k}$ is the Melweighted spectrum after passing $k^{\text {th }}$ triangular band pass filter and $L$ is the order of cepstrum. We have used order 8 that mean $L=8$.

\subsubsection{Spectral Centroid}

The spectral centroid is defined as a weighted mean of signal frequencies. It is determined by a Fourier transform using frequencies magnitudes as weights.

$$
\text { SpectralCentroid }=\frac{\sum_{n=0}^{N-1} f(n) x(n)}{\sum_{n=0}^{N-1} x(n)}
$$


Table 1: Song Segments

\begin{tabular}{|c|c|c|}
\hline Segment & Duration (second) & Type \\
\hline Intro & $40-80$ & vocal \\
\hline Bridge & $20-40$ & non-vocal \\
\hline Chorus & $20-40$ & vocal \\
\hline Verse & $60-100$ & vocal \\
\hline Outro & $40-100$ & vocal \\
\hline
\end{tabular}

where $x(n)$ is weighted frequency magnitude of bin number $n$ and $f(n)$ is center frequency of that bin.

\section{PATTERN CHECKER}

A song follows a certain pattern of vocal and non-vocal segments. Table 1 shows the different segments in a song structure with their duration. The table is based on our analysis of 74 songs found in the 10 movies we used for our experiments.

The intro of a song comes at the beginning. A verse in a song corresponds with a poetic stanza. The appearance of the verse in a song is repeated 1 to 4 times with same music but different lyrical lines. The chorus may appear many times in a song with the same lyrical lines as well as music. It is mostly sung by a group. The bridge is an interlude that connects two parts of a song. It can be played between intro and verse, between verse and outro and between repetitions of a verse. Finally, the outro is played at the end of a song. The lyrics of an outro are typically same as those of the intro, though, possibly, different in length.

The Table 1 shows that a song remains in one of two possible states: vocal and non-vocal. On other hand, the action/dialogue scenes are also composed of vocal and nonvocal segments. However a song can be differentiated from action/dialogue scenes on the basis of the duration of the vocal and non-vocal segments. For example, if a vocal appears in a song, it may be either intro, chorus, verse, or outro. The duration for these vocal segments must fall in a range between 20 to 100 seconds. On other hand, if a non-vocal segments appears in a song, it must be a bridge. Depending on the place of its appearance, the duration of the bridge in non-vocal segments ranges from 20 to 40 seconds.

The behavior of any system that remains in one of its possible states, like a song, can be simulated by an state transition system known as finite state machine. A finite state machine or finite Automaton accepts a formal language that is a set of finite string over some finite alphabet. An $\omega$-language is set of infinite words. A set of all infinite words or $\omega$ words is represented by $\Sigma^{\omega}$. An $\omega$-language is a sub set of $\Sigma^{\omega}$. An special type of automaton, $\omega$-automaton, accepts an $\omega$-language.

An $\omega$-automaton does not have a stop condition because it runs over infinite words; however, it can have a variety of acceptance condition definitions. Based on the particular acceptance condition, various types of $\omega$-automata are defined in the literature $[5,6]$. In this paper we limit our discussion to the Buchi automata [5].

A Buchi automaton is an $\omega$-automaton that accepts an $\omega$-word if there exists a run of the automaton that visits at least one of the final states infinitely often. Formally, a Buchi automaton $\mathbf{B}$ is a tuple $\left\langle\Sigma, Q, q_{s}, E, q_{f}\right\rangle$, where $\Sigma$ is a finite set containing alphabet of $\mathbf{B}, Q$ is a finite set representing the states of $\mathbf{B}$, $E \subseteq Q \times Q \times \Sigma$ is a set of edges, $q_{s}$ and $q_{f}$ represent the sets of initial and final states respectively. The automaton $\mathbf{B}$ starts in an initial state $q_{i} \in q_{s}$, and if $\left\langle q, q^{\prime}, a\right\rangle \in E$ then the automation performs a transition from the state $q$ to next state $q^{\prime}$.

Given an $\omega$-word, $\sigma=\sigma_{1} \sigma_{2} \ldots$ over some alphabet $\Sigma$, an accepting run over Buchi automaton $\mathbf{B}$ is defined as,

$$
r: q_{0} \stackrel{\sigma_{1}}{\Rightarrow} q_{1} \stackrel{\sigma_{2}}{\Rightarrow} q_{2} \stackrel{\sigma_{3}}{\Rightarrow} \ldots
$$

provided $q_{0} \in Q$, and $\left\langle q_{i-1}, q_{i}, \sigma_{i}\right\rangle \in E$ for all $i \geq 1$ and inf $f(r) \cap$ $q_{f} \neq \emptyset$ where inf $(r)$ consist of states $q \in Q$ such that $q=q_{i}$ for infinitely many $i \geq 0$. It means a run $r$ is an accepting run iff some state from $q_{f}$ is visited infinitely often along $r$.

The theory of automata is a nice way of capturing the sequence of events that may take place during the life cycle of a system. The formalisms of automata however abstracts away time and retains only the sequence of events. In the real world, though, events in a system tend to be associated with time; e.g., the correct functioning of the control system of airplanes and toasters depends crucially upon real time considerations. Thus there is a strong need of integrating timing information in finite automaton to correctly model the behavior of a real-time system.

There are many attempts in the literature to introduce the notion of a timing sequence in formal automata. In this regard we follow the pioneering work of Alur \& Dill [1]. They developed a theory of timed automata to associate timing information with a sequence of events in a system. In the following sections we give a brief explanation of timed automata and show how they can be used to model a song in our application.

\subsection{Timed automata}

Before proceeding to the actual timed automaton definition, it is important to understand the words and language that a timed automaton accepts.

\subsubsection{Time sequence}

A timing sequence $\tau=\tau_{1} \tau_{2} \ldots$ is an infinite sequence of time values $\tau_{i} \in \mathbf{R}$ with $\tau_{i}>0$, and satisfying the condition that $\tau$ increases monotonically; i.e., $\tau_{i}<\tau_{i+1}$, for all $i \geq 1$.

\subsubsection{Timed word}

A timed word is a pair $(\sigma, \tau)$ where $\sigma=\sigma_{1} \sigma_{2} \ldots$ is an infinite word over alphabet $\Sigma$ and $\tau$ is time sequence defined above. A timed word is input to the timed automaton, where $\sigma_{i}$ represents a symbol from alphabet $\Sigma$ and $\tau_{i}$ represents the time of occurrence of that input symbol.

\subsubsection{Timed language}

A Timed language over $\Sigma$ is a set of timed words over $\Sigma$.

\subsubsection{Clock and State Transition with timing con- straints}




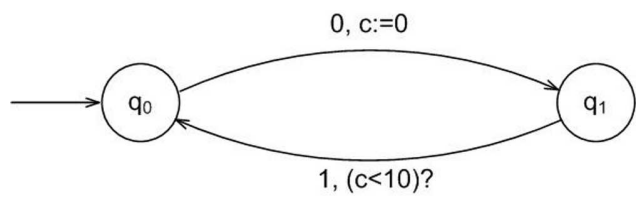

Figure 5: Timed Automaton Example

The transition in a finite automaton depends on input symbol only. In a timed automaton, the transition does not depend on the input symbol only, rather it depends on time of occurrence of that input symbol as well. In order to keep track of the timing information, a finite set of (real valued) clocks is associated with each automaton. The value of a clock can be set to 0 with any state transition. During the next state transition the value of a clock shows the time elapsed since the last time it was reset. Every state transition can be associated with clock constraints, the requirement being that a transition can take place only if the current value the clocks satisfy the constraints.

Example 5.3 Figure 5 shows a timed automaton over $\Sigma=\{0,1\}$ and $Q=\left\{q_{0}, q_{1}\right\}$ The language of this automaton is given by $L_{2}$,

$$
L_{2}=\left((01)^{\omega}, \tau\right) \mid \forall_{i} \cdot\left(\tau_{2 i}<\tau_{2 i-1}+10\right)
$$

The automaton uses a single clock $c$. The notation $c:=0$ on transition from the state $q_{0}$ to the state $q_{1}$ represents the action of resetting clock $c$. Similarly, the notation of $(c<10)$ ? represents a constraint on the edge from the state $q_{1}$ to the state $q_{0}$.

\subsubsection{Timed Automata}

A timed automaton $\mathbf{T}$ is a tuple $\left(\Sigma, \mathrm{Q}, q_{s}, \mathrm{C}, \mathrm{E}\right)$, where $\Sigma$ is a finite alphabet, $Q$ is a finite set of states, $q_{s} \subseteq Q$ is a set of start states $C$ is a finite set of clocks, and $E \subseteq S \times S \times \Sigma \times 2^{C} \times \phi(C)$ gives the set of transitions. An edge $\left(q, q^{\prime}, a, \lambda, \delta\right)$ represents a transition from state $q$ to state $q^{\prime}$ on input symbol $a$. The set $\lambda \subseteq C$ gives the clocks to be reset with this transition, and $\delta$ is clock constraint over $C$.

Given a timed word $(\sigma, \tau)$ the timed automaton starts in one of its starting states at the time 0 with all its clocks initialized to 0 . As time advances, the value of all the clocks change, reflecting the elapsed time. At the time $\tau_{i}$, the automaton changes state from $q$ to $q^{\prime}$ using some transition of the form $\left(q, q^{\prime}, \sigma_{i}, \lambda, \delta\right)$ reading the input symbol $\sigma_{i}$, if the current value of clocks satisfy $\delta$. With this transition the clocks in $\lambda$ are reset to 0 , and thus start counting time again with respect to the time of occurrence of this transition.

\subsubsection{Run of Timed Automata}

A run $r$, denoted by $(q, v)$ of a timed automaton $\left(\Sigma, Q, q_{0}, C, E\right)$ over a timed word $(\sigma, \tau)$ is an infinite sequence of the form,

$$
r:\left(q_{0}, v_{0}\right) \underset{\tau_{1}}{\stackrel{\sigma_{1}}{\Rightarrow}}\left(q_{1}, v_{1}\right) \underset{\tau_{2}}{\stackrel{\sigma_{2}}{\Rightarrow}}\left(q_{2}, v_{2}\right) \underset{\tau_{2}}{\stackrel{\sigma_{2}}{\Rightarrow}} \ldots
$$

with $q_{i} \in Q$ and $v_{i} \in[C \rightarrow \mathbf{R}]$, for all $i \geq 0$.

\subsection{Probabilistic Timed Automata}

The probabilistic timed automata are extensions of timed automata where each transition along with timing constraints,
Table 2: Song Timed Words

\begin{tabular}{|l|l|}
\hline Song Title & Timed Word \\
\hline iss se pehley & $\begin{array}{l}(\mathrm{NV}, 1)(\mathrm{V}, 23)(\mathrm{NV}, 74)(\mathrm{V}, 102) \\
(\mathrm{NV}, 182)(\mathrm{V}, 198)(\mathrm{NV}, 242)(\mathrm{V}, 269)\end{array}$ \\
\hline pal do pal & $(\mathrm{NV}, 1)(\mathrm{V}, 19)(\mathrm{NV}, 102)(\mathrm{V}, 127)$ \\
& $(\mathrm{NV}, 202)(\mathrm{V}, 218)(\mathrm{NV}, 238)(\mathrm{V}, 247)$ \\
& $(\mathrm{NV}, 301)$ \\
\hline koi lota de & $(\mathrm{NV}, 1)(\mathrm{V}, 21)(\mathrm{NV}, 107)(\mathrm{V}, 119)$ \\
& $(\mathrm{NV}, 165)(\mathrm{V}, 187)(\mathrm{NV}, 250)(\mathrm{V}, 271)$ \\
\hline kaise din jeevan & $(\mathrm{NV}, 1)(\mathrm{V}, 13)(\mathrm{NV}, 73)(\mathrm{V}, 91)$ \\
& $(\mathrm{NV}, 160)(\mathrm{V}, 181)(\mathrm{NV}, 209)(\mathrm{V}, 229)$ \\
& $(\mathrm{NV}, 299)$ \\
\hline main rahon na & $(\mathrm{NV}, 1)(\mathrm{V}, 33)(\mathrm{NV}, 101)(\mathrm{V}, 127)$ \\
& $(\mathrm{NV}, 188)(\mathrm{V}, 202)(\mathrm{NV}, 237)(\mathrm{V}, 261)$ \\
\hline
\end{tabular}

Table 3: Dialogue Timed Words

\begin{tabular}{|l|l|}
\hline Dialogue No. & Timed Word \\
\hline Dialogue 1 & $(\mathrm{NV}, 6)(\mathrm{V}, 7)(\mathrm{NV}, 95)(\mathrm{V}, 101)(\mathrm{NV}, 159)$ \\
\hline Dialogue 2 & $(\mathrm{NV}, 8)(\mathrm{V}, 9)(\mathrm{NV}, 22)(\mathrm{V}, 24)$ \\
& $(\mathrm{NV}, 28)(\mathrm{V}, 33)$ \\
\hline Dialogue 3 & $(\mathrm{NV}, 4)(\mathrm{V}, 5)(\mathrm{NV}, 10)(\mathrm{V}, 12)$ \\
& $(\mathrm{NV}, 15)(\mathrm{V}, 18)(\mathrm{NV}, 60)(\mathrm{V}, 62)$ \\
& $(\mathrm{NV}, 92)(\mathrm{V}, 94)(\mathrm{NV}, 101)(\mathrm{V}, 110)$ \\
& $(\mathrm{NV}, 117)(\mathrm{V}, 119)(\mathrm{NV}, 126)(\mathrm{V}, 128)$ \\
& $(\mathrm{NV}, 223)(\mathrm{V}, 231)(\mathrm{NV}, 247)(\mathrm{V}, 249)$ \\
\hline Dialogue 4 & $(\mathrm{NV}, 17)(\mathrm{V}, 38)(\mathrm{NV}, 52)(\mathrm{V}, 57)$ \\
& $(\mathrm{NV}, 64)(\mathrm{V}, 71)(\mathrm{NV}, 73)(\mathrm{V}, 84)$ \\
& $(\mathrm{NV}, 90)(\mathrm{V}, 117)(\mathrm{NV}, 123)(\mathrm{V}, 125)$ \\
& $(\mathrm{NV}, 128)(\mathrm{V}, 142)(\mathrm{NV}, 144)(\mathrm{V}, 146)$ \\
& $(\mathrm{NV}, 148)(\mathrm{V}, 150)(\mathrm{NV}, 167)(\mathrm{V}, 169)$ \\
\hline Dialogue 5 & $(\mathrm{NV}, 4)(\mathrm{V}, 7)(\mathrm{NV}, 57)(\mathrm{V}, 60)$ \\
& $(\mathrm{NV}, 76)(\mathrm{V}, 80)(\mathrm{NV}, 86)(\mathrm{V}, 96)$ \\
& $(\mathrm{NV}, 99)(\mathrm{V}, 102)(\mathrm{NV}, 105)(\mathrm{V}, 108)$ \\
& $(\mathrm{NV}, 110)(\mathrm{V}, 113)(\mathrm{NV}, 116)(\mathrm{V}, 119)$ \\
\hline
\end{tabular}

contains a probability value as well. The probability value on an edge between two states $q$ and $q^{\prime}$ represents the chance of transition given a symbol $a$. The probability value 0 between two states represents that there is no edge between two states. Formally a probabilistic timed automaton $\mathbf{T}$ is a tuple $\left(\Sigma, Q, q_{s}, C, E, \operatorname{Pr}\right)$ where, $\Sigma$ is finite alphabet, $Q$ is finite set of states, $q_{s} \subseteq S$ is set of start state, $C$ is finite set of clocks, $E \subseteq S \times S \times \Sigma \times 2^{C} \times \phi(C)$ gives the set of transitions. An edge $\left(s, s^{\prime}, a, \lambda, \delta\right)$ represents a transition from state $s$ to state $s^{\prime}$ on input symbol $a$. The set $\delta \subseteq C$ gives the clocks to be reset with this transition, $\lambda$ represents clock constraints over set $C$, and $\operatorname{Pr}: \Sigma \times E \rightarrow[0,1]$ represents a probability transition function such that for all $q \in Q$ and $a \in \Sigma$ where $\sum_{e \in E} p(a, e)=1$ represents probability to use edge from state $q$ to state $q^{\prime}$ if symbol $a$ is read.

\subsection{Probabilistic Timed Automaton for PSS Clas- sification}

A potential song sequence is an audio/video sequence which may or may not be a song as explained in section 4 . If a potential song sequence contains either an action or dialogue scene, it should be discarded. We accomplish this by classifying potential song sequences in two classes: song and nonSong. 
Table 4: Song State Transition

\begin{tabular}{|l|l|}
\hline Song Title & State Transition \\
\hline iss se pehley & $q_{0} \rightarrow q_{1} \rightarrow q_{2} \rightarrow q_{1} \rightarrow q_{2} \rightarrow q_{1} \rightarrow q_{3} \rightarrow$ \\
& $q_{1} \rightarrow q_{2}$ \\
\hline pal do pal & $q_{0} \rightarrow q_{1} \rightarrow q_{3} \rightarrow q_{1} \rightarrow q_{2} \rightarrow q_{1} \rightarrow q_{3} \rightarrow$ \\
& $q_{1} \rightarrow q_{3} \rightarrow q_{1}$ \\
\hline koi lota de & $q_{0} \rightarrow q_{1} \rightarrow q_{2} \rightarrow q_{1} \rightarrow q_{3} \rightarrow q_{1} \rightarrow q_{2} \rightarrow$ \\
& $q_{1} \rightarrow q_{2}$ \\
\hline kaise din jeevan & $q_{0} \rightarrow q_{1} \rightarrow q_{3} \rightarrow q_{1} \rightarrow q_{2} \rightarrow q_{1} \rightarrow q_{2} \rightarrow$ \\
& $q_{1} \rightarrow q_{2} \rightarrow q_{1}$ \\
\hline main rahon na & $q_{0} \rightarrow q_{1} \rightarrow q_{2} \rightarrow q_{1} \rightarrow q_{2} \rightarrow q_{1} \rightarrow q_{3} \rightarrow$ \\
& $q_{1} \rightarrow q_{2}$ \\
\hline
\end{tabular}

Table 5: Action/Dialogue State Transition

\begin{tabular}{|l|l|}
\hline Song Title & State Transition \\
\hline Dialogue 1 & $q_{0} \rightarrow q_{1} \rightarrow q_{3} \rightarrow q_{1} \rightarrow q_{3} \rightarrow q_{1}$ \\
\hline Dialogue 2 & $q_{0} \rightarrow q_{1} \rightarrow q_{3} \rightarrow q_{4} \rightarrow q_{3} \rightarrow q_{4} \rightarrow q_{3}$ \\
\hline Dialogue 3 & $q_{0} \rightarrow q_{1} \rightarrow q_{3} \rightarrow q_{4} \rightarrow q_{3} \rightarrow q_{4} \rightarrow q_{3} \rightarrow$ \\
& $q_{1} \rightarrow q_{3} \rightarrow q_{1} \rightarrow q_{3} \rightarrow q_{4} \rightarrow q_{3} \rightarrow q_{4} \rightarrow$ \\
& $q_{3} \rightarrow q_{4} \rightarrow q_{3} \rightarrow q_{4} \rightarrow q_{3} \rightarrow q_{4} \rightarrow q_{3}$ \\
\hline Dialogue 4 & $q_{0} \rightarrow q_{1} \rightarrow q_{2} \rightarrow q_{4} \rightarrow q_{3} \rightarrow q_{4} \rightarrow q_{3} \rightarrow$ \\
& $q_{4} \rightarrow q_{3} \rightarrow q_{4} \rightarrow q_{2} \rightarrow q_{4} \rightarrow q_{3} \rightarrow q_{4} \rightarrow$ \\
& $q_{3} \rightarrow q_{4} \rightarrow q_{3} \rightarrow q_{4} \rightarrow q_{3} \rightarrow q_{4} \rightarrow q_{3}$ \\
\hline Dialogue 5 & $q_{0} \rightarrow q_{1} \rightarrow q_{3} \rightarrow q_{1} \rightarrow q_{3} \rightarrow q_{4} \rightarrow q_{3} \rightarrow$ \\
& $q_{4} \rightarrow q_{3} \rightarrow q_{4} \rightarrow q_{3} \rightarrow q_{4} \rightarrow q_{3} \rightarrow q_{4} \rightarrow$ \\
& $q_{3} \rightarrow q_{4} \rightarrow q_{3}$ \\
\hline
\end{tabular}

In this section, we develop a probabilistic timed automaton that can differentiate between an action/dialogue PSS from a song PSS. The basic difference between a PSS representing a song and a PSS representing action/dialogue scene is their vocal/non-vocal composition. The vocal segments length in a PSS representing a song should be between 20 to 100 seconds whereas for the non-vocal, it should be between 20 to 40 seconds as observed from different segments of song shown in Table 1. On other hand, such durations were observed to be completely random and mostly falling outside these ranges in PSS representing action/dialogue scenes.

Based on the duration assumptions of the vocal and non-vocal segments, we developed a probabilistic timed automaton to classify a PSS in song and non-song classes. The PSSs classified in a song class are output of the system, whereas the PSSs classified in non-song class are discarded. The Figure 6 show the state transitions of this probabilistic timed automaton. The alphabet of the automaton is $\Sigma=\{V, N V\}$, the set of states is $Q=\left\{q_{0}, q_{1}, q_{2}, q_{3}, q_{4}\right\}$, with initial state $q_{s}=\left\{q_{0}\right\}$, and the set of clocks is defined as $C=\{X\}$.

\subsubsection{Probabilistic Timed Automaton Classifier: Train- ing Phase}

In training phase of a probabilistic timed automaton used for the classification, we need to calculate the state transition probabilities for different classes. In this section we show the process of calculating these probabilities.

We experimented with 30 songs and 30 action/dialogue potential song sequences to generate probability values for the state transition of Figure 6. Every PSS generated by the PSSG

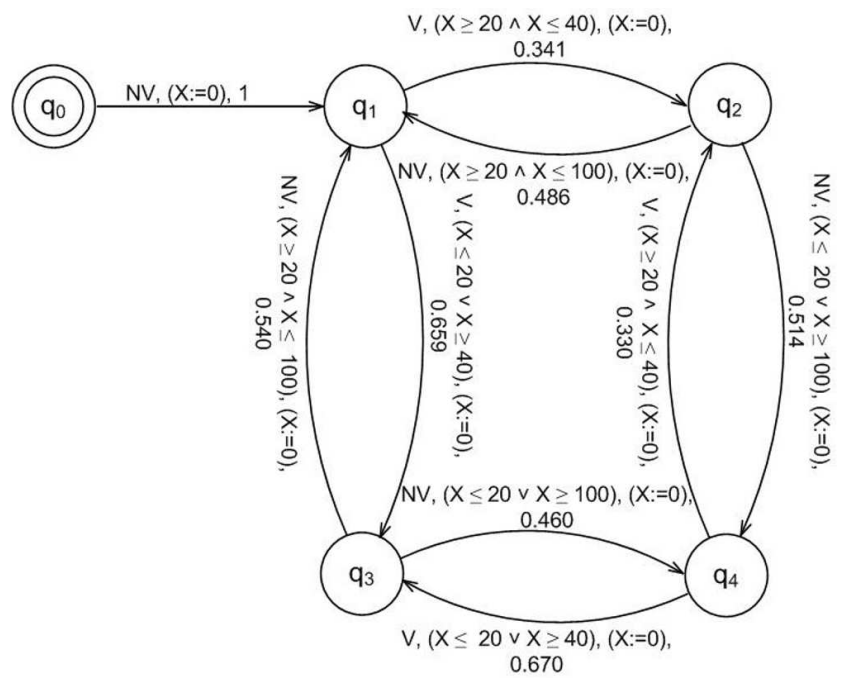

Figure 6: Probabilistic Timed Automaton for Song

is converted into a timed word, where each timed word shows the appearance of either vocal or non-vocal segment with its time. For example, a timed word $(V, 10),(N V, 30)$ represents the appearance of $V$ (vocal) segments at $10^{\text {th }}$ second and $N V$ (non-vocal) at $30^{\text {th }}$ second. Due to space limitation, we give here only 5 samples of timed word each from the song and action/dialogue PSS as shown in Table 2 and Table 3 respectively. The sample timed words shown in Table 2 and Table 3 were applied to the timed automaton of Figure 6. The resulting state transitions for song and non-song are shown in Table 4 and Table 5. We estimate the probabilities of the state transition for the song and the action/dialogue from Table 4 and Table 5 respectively. The Figure 6 and Figure 7 show the probabilistic timed automaton for the song and action/dialogue with their state transition probabilities derived from their timed words shown in Table 2 and Table 3. The state transition of a probabilistic timed automaton can also be represented in a matrix form. The matrices song and nonSong show the state transition of the song and non-song (action/dialogue) classes for their respective models shown in Figure 6 and Figure 7.

$$
\text { song }=\left[\begin{array}{cccccc} 
& q_{0} & q_{1} & q_{2} & q_{3} & q_{4} \\
q_{0} & 0 & 1 & 0 & 0 & 0 \\
q_{1} & 0 & 0 & 0.341 & 0.659 & 0 \\
q_{2} & 0 & 0.486 & 0 & 0 & 0.514 \\
q_{3} & 0 & 0.540 & 0 & 0 & 0.460 \\
q_{4} & 0 & 0 & 0.330 & 0.670 & 0
\end{array}\right]
$$

$$
\text { nonSong }=\left[\begin{array}{cccccc} 
& q_{0} & q_{1} & q_{2} & q_{3} & q_{4} \\
q_{0} & 0 & 1 & 0 & 0 & 0 \\
q_{1} & 0 & 0 & 0.254 & 0.746 & 0 \\
q_{2} & 0 & 0.404 & 0 & 0 & 0.596 \\
q_{3} & 0 & 0.532 & 0 & 0 & 0.468 \\
q_{4} & 0 & 0 & 0.281 & 0.719 & 0
\end{array}\right]
$$

\subsubsection{Probabilistic Timed Automaton Classifier: Test Phase}




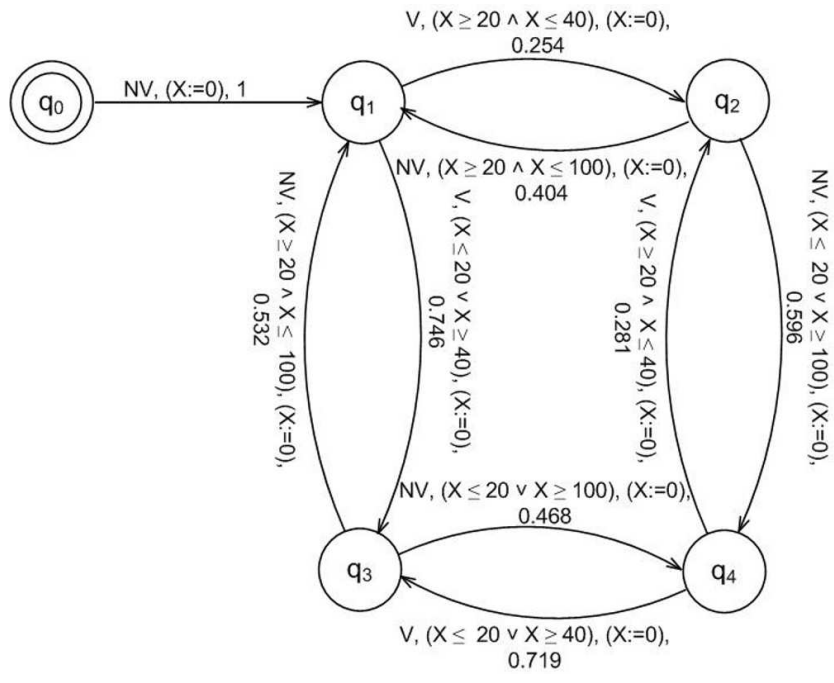

Figure 7: Probabilistic Timed Automaton for Non-Song

During the test phase, we break an input potential song sequence $P S S$ in a timed word $T$ defined as,

$$
T=(\sigma, \tau)
$$

where $\sigma=\sigma_{1} \sigma_{2} \ldots$ is an infinite word over alphabet $\Sigma=\{V, N V\}$ and $\tau$ represents the time of occurrence of that word.

The next step is to run timed word T over the automaton of Figure 6 to derive the state transition $S=q_{0} \rightarrow q_{1} \rightarrow q_{2} \ldots q_{L}$ of length $L$. In order to classify the resultant state transition $S$ of length $L$ in either song or nonSong model, we compute the weights $w_{1}$ and $w_{2}$, defined as,

$$
\begin{gathered}
w_{1}=\prod_{i=1}^{L} \operatorname{Tr}_{\text {song }}\left(q_{i j}\right) \\
w_{2}=\prod_{i=1}^{L} \operatorname{Tr} r_{\text {nonsong }}\left(q_{i j}\right)
\end{gathered}
$$

where:

$\operatorname{Tr}_{\text {song }}\left(q_{i j}\right) \rightarrow$ Probability value of transition from state $q_{i}$ to state $q_{j}$ in song matrix.

$\operatorname{Tr}_{\text {nonsong }}\left(q_{i j}\right) \rightarrow$ Probability value transition from state $q_{i}$ to state $q_{j}$ in nonSong model.

Finally, given a potential song sequence PSS,

$$
\text { PSS }= \begin{cases}\text { song } & w_{1} \geq w_{2} \\ \text { action } / \text { dialogue } & w_{1}<w_{2} .\end{cases}
$$

Example 5.4 (Song and nonSong classification) Suppose we have a potential song sequence that results in following timed word,

\section{$(N V, 1)(V, 13)(N V, 31)(V, 53)(N V, 70)(V, 91)(N V, 140)(V, 163)$ $(N V, 202)(V, 227) \ldots$}

It shows that there is start of non-vocal (NV) at second 1 followed by vocal $(\mathrm{V})$ at second 13 . The next NV is found at second 31 and so on. When we apply this to our state transition model of the Figure 6, following is the run of this timed word,

$$
\begin{aligned}
& \left(q_{0},[0]\right) \underset{1}{\stackrel{N V}{\rightleftharpoons}}\left(q_{1},[0]\right) \underset{8}{\stackrel{V}{\Rightarrow}}\left(q_{3},[0]\right) \underset{13}{\stackrel{N V}{\rightleftharpoons}}\left(q_{4},[0]\right) \underset{30}{\stackrel{V}{\Rightarrow}}\left(q_{2},[0]\right) \underset{33}{\stackrel{N V}{\rightleftharpoons}} \\
& \left(q_{4},[0]\right) \underset{51}{\stackrel{V}{\Rightarrow}}\left(q_{2},[0]\right) \underset{72}{\stackrel{N V}{\rightleftharpoons}}\left(q_{1},[0]\right) \underset{98}{\stackrel{V}{\Rightarrow}}\left(q_{2},[0]\right) \underset{112}{\stackrel{N V}{\rightleftharpoons}}\left(q_{1},[0]\right) \underset{138}{\stackrel{V}{\rightleftharpoons}} \\
& \left(q_{2},[0]\right)
\end{aligned}
$$

Thus the resultant state transition for the given word is,

$$
q_{0} \rightarrow q_{1} \rightarrow q_{3} \rightarrow q_{4} \rightarrow q_{2} \rightarrow q_{4} \rightarrow q_{2} \rightarrow q_{1} \rightarrow q_{2} \rightarrow q_{1} \rightarrow q_{2}
$$

Now we use matrices song and nonSong to calculate $w_{1}$ and $w_{2}$ respectively.

$$
w_{1}=\mathbf{0 . 0 0 0 2 4 1}
$$

and,

$$
w_{2}=\mathbf{0 . 0 0 0 1 7 3}
$$

The result shows that the value of $w_{1}$ is greater than $w_{2}$, thus the given input sequence of this example would be classified as a song.

\section{RESULTS}

We experimented with 10 popular Bollywood movies to test the proposed system of song extraction. Movies were selected over the period 1967 to 2010 and of diverse movie genres. The Table 6 shows our experimental results on selected movies. The column Potential Song Sequences Extracted in this table shows the output at the stage of Potential Song Sequences Generator. A PSS may or may not contain a song, thus in order to discard unwanted PSS that may represent an action or dialogue scene, we apply it to pattern checker. The elimination of such PSS by pattern checker improves systems precision. The output from pattern checker is shown in column Song Extracted. We define our results in precision and recall as defined below,

$$
\begin{aligned}
& \text { Precision }=\frac{\text { No. of Correctly Extracted Songs }}{\text { Total Songs Extracted }} \times 100 \\
& \text { Recall }=\frac{\text { No. of Correctly Extracted Songs }}{\text { Actual Number of Songs in Movies }} \times 100
\end{aligned}
$$

The achieved values for precision and recall were $87.34 \%$ and $93.24 \%$ respectively.

\section{CONCLUSIONS}

The Indian film industry, particularly the segment of the industry, commonly known as Bollywood, which produces Hindi language movies, enjoys a wide following in South Asia, the Middle East, Central Asia, North Africa and in countries, such as the UK and USA, where there is a significant presence of the South Asian diaspora. It produces the highest number of movies in world yearly. Music and songs contribute heavily to the success of a movie and the revenue it generates. There are, typically, five to ten songs, each of three to ten minutes, in every Bollywood movie of two to three hours. Music lovers around the world like to listen songs of Bollywood movies. However, locating their favorite song from a movie of two to three hours is a tedious task. In this paper, we have explained our ongoing work on an automatic song extractor. Such a song extractor can also 
Table 6: Song Extraction Results

\begin{tabular}{|c|c|c|c|c|c|c|c|c|}
\hline Movie & Genre & $\begin{array}{c}\text { Release } \\
\text { Year }\end{array}$ & $\begin{array}{l}\text { No. of } \\
\text { Songs }\end{array}$ & $\begin{array}{l}\text { Potential Song } \\
\text { Sequences } \\
\text { Extracted }\end{array}$ & $\begin{array}{c}\text { Song } \\
\text { Extracted }\end{array}$ & $\begin{array}{c}\text { Song } \\
\text { Missed }\end{array}$ & Precision (\%) & Recall (\%) \\
\hline Raaz & Drama & 1967 & 7 & 9 & 6 & 1 & 100 & 85.71 \\
\hline Zameer & Drama & 1975 & 7 & 11 & 7 & 0 & 100 & 100 \\
\hline Shaan & Action & 1980 & 6 & 9 & 6 & 0 & 100 & 100 \\
\hline Hum dono & Love Story & 1985 & 5 & 5 & 5 & 0 & 100 & 100 \\
\hline Dil & Love Story & 1990 & 6 & 8 & 6 & 0 & 100 & 100 \\
\hline Bewafa sanam & Love Story & 1995 & 11 & 12 & 11 & 2 & 81.82 & 81.82 \\
\hline Kasoor & Drama & 2000 & 6 & 8 & 7 & 0 & 85.71 & 100 \\
\hline No Entry & Comedy & 2005 & 8 & 11 & 10 & 0 & 80 & 100 \\
\hline Dabang & Action & 2010 & 10 & 16 & 12 & 1 & 75 & 90 \\
\hline Golmaal 3 & Comedy & 2010 & 8 & 12 & 9 & 1 & 77.78 & 87.5 \\
\hline \multicolumn{3}{|c|}{ Total } & 74 & 101 & 79 & 5 & 87.34 & 93.24 \\
\hline
\end{tabular}

assist in production of $\mathrm{CD} /$ cassettes that contains songs from different movies.

The proposed song extractor first classifies the input movie into musical and non-musical segments. It then utilizes a song grammar to construct a probabilistic timed automaton to locate songs in the musical segements. The system has been tested on ten popular Bollywood movies of diverse genres from a 40-year period. The selected movies contain a total of 74 songs out of which 69 were successfully extracted, thus achieving a recall as good as $93.24 \%$. The precision value of the proposed system on experimental data was found to be $87.34 \%$.

\section{REFERENCES}

[1] R. Alur and D. Dill. A theory of timed automata. Theoretical Computer Science, 126:183-235, 1994.

[2] H. L. Bart Lehane, Noel E. OConnor and A. F. Smeaton. Indexing of fictional video content for event detection and summarization. Journal on Image and Video Processing: Special Issue on Multimodal Audiovisual Content Abstraction, 2007(2):1-15, August 2007.

[3] N. O. C. Bart Lehane and N. Murphy. Dialogue scene detection in movies using low and mid-level visual features. In Proceeding of International Workshop on Image, Video, and Audio Retrieval and Mining," pages 25-35. Quebec, Canada, October 2004.

[4] B. Bhavan. Central Board Of Film Certification: Annual Report. Ministry of Information and Broadcasting, Government of India, 91-E, Walkeshwar Road, Mumbai, India, 2009.

[5] R. Buchi. On a decision method in restricted second-order arithmetic. In Proceedings of Internat. Cong. on Logic, Methodology, and Philosophy of Science, pages 1-12, May 1962.

[6] Y. Choueka. Theories of automata on omega-tapes: A simplified approach. Journal of Computer and System Sciences, 8:117-141, 1974.

[7] M. S. Eric Scheirer. Construction and evaluation of robust multifeature speech/music discriminator. In Proceedings of the 1997 IEEE International Conference on Acoustics, Speech, and Signal Processing, pages 1331-1334. Washington, DC, USA, April 1997.

[8] J. y. A. Hadi Harb, Liming Chen. Speech/music/silence and gender detection algorithm. In Proceedings of the 7th International conference on Distributed Multimedia Systems, pages 257-262, September 1997.

[9] H. jiang Zhang, A. Kankanhalli, S. W. Smoliar, and S. Tan. Automatic partitioning of full-motion video. Multimedia Systems, 1:10-28, 1993.

[10] S. J. R. Lei Chen and M. T. Ozsu. Incorporating audio cues into dialog and action scene extraction. In Proceeding of Storage and Retrieval for Media Databases,, pages 352-363. Santa Clara, CA, USA (2003), September 2003.

[11] D. Li, I. K. Sethi, N. Dimitrova, and T. Mcgee. Classification of general audio data for content-based retrieval. Pattern Recognition Letters, 22:533-544, 2001.

[12] H. j. Z. Lie Lu and H. Jiang. Content analysis for audio classification and segmentation. IEEE Transactions on Audio, Speech and Language Processing, 10:504-516, 2002.

[13] H.-J. Z. Lie Lu, Stan Z. Li. Content-based audio segmentation using support vector machines. In Proceedings IEEE International Conference on Multimedia and Expo, 2001., pages 749-752, October 2001.

[14] L. Lu, H. J. Zhang, and S. Z. Li. Content-based audio classification and segmentation by using support vector machines. Multimedia Systems, 8:482-492, 2003.

[15] C. Panagiotakis and G. Tziritas. A speech/music discriminator based on rms and zero-crossings. IEEE Transactions on Multimedia, 7:155-166, 2005.

[16] J. Saunders. Real time discrimination of broadcast speech/music. In International Conference on Acoustics, Speech, and Signal Processing, 1996. 